\title{
Loss of CIp2r signaling activates hepatic stellate cells and exacerbates diet-induced steatohepatitis in mice
}

\author{
Shai Fuchs,,$^{1,2}$ Bernardo Yusta, ${ }^{1}$ Laurie L. Baggio, ${ }^{1}$ Elodie M. Varin, ${ }^{1}$ Dianne Matthews, ${ }^{1}$ \\ and Daniel J. Drucker ${ }^{1,3}$ \\ ${ }^{1}$ Lunenfeld Tanenbaum Research Institute, Mount Sinai Hospital, Toronto, Ontario, Canada. ${ }^{2}$ The Hospital for Sick Children \\ and ${ }^{3}$ Department of Medicine, University of Toronto, Toronto, Ontario, Canada.
}

A glucagon-like peptide-2 (CLP-2) analog is used in individuals with intestinal failure who are at risk for liver disease, yet the hepatic actions of GLP-2 are not understood. Treatment of highfat diet-fed (HFD-fed) mice with CLP-2 did not modify the development of hepatosteatosis or hepatic inflammation. In contrast, Glp2 $r^{-1-}$ mice exhibited increased hepatic lipid accumulation, deterioration in glucose tolerance, and upregulation of biomarkers of hepatic inflammation. Both mouse and human liver expressed the canonical GLP-2 receptor (CLP-2R), and hepatic GIp2r expression was upregulated in mice with hepatosteatosis. Cell fractionation localized the GIp2r to hepatic stellate cells (HSCs), and markers of HSC activation and fibrosis were increased in livers of $\mathrm{Clp} 2 \mathrm{r}^{-/-}$mice. Moreover, GLP-2 directly modulated gene expression in isolated HSCs ex vivo. Taken together, these findings define an essential role for the GLP-2R in hepatic adaptation to nutrient excess and unveil a gut hormone-HSC axis, linking GLP-2R signaling to control of HSC activation.

\section{Conflict of interest: The}

pharmaceutical development of GLP-2 is the subject of a license agreement among DJD, Toronto General Hospital, the University of Toronto, and ShireTakeda Inc. This work was supported in part by an investigator-initiated operating grant from Shire-Takeda Inc. to Mt. Sinai Hospital for work in the Ducker laboratory.

Copyright: (c) 2020, American Society for Clinical Investigation.

Submitted: January 30, 2020

Accepted: March 12, 2020

Published: April 23, 2020

Reference information: /CI Insight. 2020;5(8):e136907.

https://doi.org/10.1172/jci.

insight.136907.

\section{Introduction}

Glucagon-like peptide-2 (GLP-2) is a 33-amino acid proglucagon-derived peptide that is secreted, together with GLP-1, from the intestinal enteroendocrine L cell (1). GLP-2 is continuously secreted at low basal levels in the fasting or interprandial state, and circulating levels rise briskly within minutes of food ingestion (2). GLP-2 exerts proliferative and cytoprotective actions in the small bowel, resulting in the expansion of the mucosal surface area and enhanced nutrient absorption $(3,4)$. Sustained augmentation of GLP-2R signaling with a degradation-resistant GLP-2 analog, teduglutide, reduces the requirement for parenteral nutrition (PN) in human subjects with short-bowel syndrome (SBS) (5), and teduglutide is approved for the therapy of adults and children with PN-dependent SBS (6).

Although the actions of GLP-2 are predominantly localized to the gut (7), the extraintestinal expression of the GLP-2R raises important questions surrounding the safety of therapy with GLP-2R agonists, and potential actions of teduglutide beyond the gastrointestinal tract. For example, we recently detected robust expression of the GLP-2R in gallbladder and demonstrated that administration of teduglutide markedly inhibits gallbladder emptying in mice (8). These findings, subsequently extended to human studies, are consistent with reports of increased numbers of gallbladder-related adverse events associated with teduglutide therapy (9).

A full-length GLP-2 receptor (GLP-2R) mRNA transcript has also been identified in murine liver, albeit at levels much lower than in the jejunum (10). The GLP-2R was localized to hepatocytes (11); however, GLP-2R mRNA transcripts were not identified within hepatocytes profiled by single-cell RNA sequencing (12). Moreover, the biological actions of GLP-2 on the liver are conflicting, with some preclinical studies demonstrating a detrimental effect on hepatic steatosis (13), whereas other reports highlight a hepatoprotective role for GLP-2, characterized by a reduction in markers of systemic and hepatic inflammation (14). The frequent development of hepatic steatosis in PN-dependent subjects with SBS, associated in some subjects with progression to hepatic failure and liver transplantation, highlights the importance of understanding the liver as a target of GLP-2 action $(15,16)$.

To resolve the hepatic actions of GLP-2, we have now studied GLP-2 action in high-fat, high-fructose, high-cholesterol-fed (HFHC-fed) WT mice and simultaneously examined the hepatic phenotype of Glp $2 r^{-1}$ mice. Our findings demonstrate that expression of the hepatic Glp2r is induced following high-fat 
diet (HFD) feeding; however, sustained GLP-2R agonism has little effect on the development of hepatic steatosis. In contrast, loss of the GLP-2R increases steatosis and biomarkers of hepatic inflammation. Furthermore, we localize a functional GLP-2R to hepatic stellate cells (HSCs) and demonstrate increased markers of stellate cell activation in $G l p 2 r^{-1}$ mice. Collectively, these findings reveal a prandial signal transduction pathway linking gut-derived GLP-2 to the control of HSC activity, with implications for understanding GLP-2 action in the context of nutrition-associated hepatocellular injury.

\section{Results}

GLP-2 does not alter the metabolic and inflammatory sequelae of HFHC feeding. Previous studies of metabolic and hepatic health in HFD-fed mice and rats treated with GLP-2 have yielded conflicting results $(13,17)$. To study the effect of GLP-2 in the setting of energy excess, we administered GLP-2 to mice on (a) a HFHC diet or (b) a 10\% fat control diet (CD) matched for other macronutrient sources. On week 15, mice were randomly allocated to treatment with either the long-acting GLP-2 analog h[Gly2]GLP-2, which is identical to teduglutide (hereafter referred to as GLP-2), or saline, for 11 days (Figure 1A). Body and liver weight were increased by the HFHC diet but were not different in GLP-2-treated mice (Figure 1B and Supplemental Figure 1A; supplemental material available online with this article; https://doi.org/10.1172/ jci.insight.136907DS1). GLP-2 increased small bowel length, total intestinal weight, and weight per $\mathrm{cm}$ as well as gallbladder volume (Supplemental Figure 1, B and C). Glucose tolerance (i.p.) and plasma lipid profiles (LDL, HDL, total cholesterol, and triglyceride [TG]) were unaffected by GLP-2 treatment (Figure 1, C-E). Hepatic Oil Red O staining (Figure 1F) and hepatic cholesterol and TG content (Figure 1G) were increased after exposure to HFHC diet but not different in GLP-2-treated mice. Levels of hepatic mRNA transcripts encoding proteins important for lipid metabolic pathways were frequently modulated by diet but not altered by GLP-2 treatment (Figure $1 \mathrm{H}$ ).

Plasma alanine transferase (ALT) levels were higher (Figure 2A) and hepatic and plasma levels of KC/ GRO, TNF- $\alpha$, and IL-10 were elevated in HFHC-fed mice; however, none of the measured hepatic or plasma cytokines, including IL-1 $\beta$, IFN- $\gamma$, IL-6, and IL-5, were different in GLP-2-treated mice (Figure 2B and Supplemental Figure 1D). Similarly, hepatic mRNA transcripts corresponding to markers of inflammation and fibrosis were not different after GLP-2 administration (Figure 2C). Thus, in HFHC diet-induced steatohepatitis, GLP-2 treatment did not impair indices of glucose and lipid metabolism or hepatic and systemic inflammation.

Glp2r-1 mice exhibit impaired glucose tolerance and enhanced hepatic fat deposition. Previous studies have demonstrated that both gain and loss of GLP-2 action promoted hepatic steatosis in HFD-fed mice (13, 17). To resolve this discrepancy, we assessed indices of glucose and lipid metabolism in male $G l p 2 r^{-1}$ and Glp2 $r^{+/+}$littermate control mice fed a HFHC or CD for 17 weeks (Figure 3A). HFHC-fed mice exhibited increased weight gain and liver mass; however, these parameters were not different in $G l p 2 r^{-1}$ mice (Figure 3B and Supplemental Figure 2A). Glp2 $r^{-1-}$ mice demonstrated elevated AUC glucose during the second hour of i.p. glucose tolerance (Figure 3C). Fasting plasma lipid profiles, including LDL, HDL, total cholesterol, and TG, were not different in Glp2r/- versus $\mathrm{Glp}_{2 r^{+/+}}$mice (Figure 3, D and E).

CD-fed Glp2r $\mathrm{r}^{-1}$ mice exhibited greater hepatic Oil Red O staining (Figure 3F) and hepatic cholesterol and TG content were elevated in HFHC diet-fed mice, with no difference between genotypes. However, hepatic cholesterol and TG levels were increased in CD-fed Glp2r/- versus Glp2r+/+ mice, despite similar body weights in both genotypes (Figure 3, B and G). We next assessed levels of mRNA transcripts corresponding to genes important for TG and cholesterol uptake and degradation and de novo lipogenesis. Although the majority of hepatic mRNA transcript levels were not different between genotypes, levels of

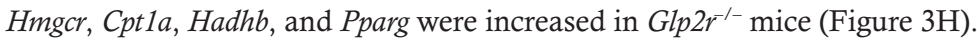

To assess extrahepatic mechanisms that could modify the extent of hepatic lipid deposition in Glp2r-1 mice, we studied oral lipid absorption as well as dipeptidyl peptidase-4 (DPP-4) activity and prandial GLP1 levels in a separate cohort of mice. Plasma DPP-4 activity was not different (Supplemental Figure 2C), whereas fasting GLP-1 levels trended higher in Glp2r-- mice $(P=0.07)$ (Supplemental Figure 2D). Plasma TGs were not different in the fasting state or after olive oil challenge (Supplemental Figure 2, E and F); however, plasma GLP-1 levels were higher in Glp2 $r^{\prime-}$ mice after enteral olive oil administration (Supplemental Figure 2G). Accordingly, putative differences in DPP-4 activity and increased GLP-1 levels, which might also reduce postprandial lipemia $(18,19)$, do not likely contribute to elevated lipid deposition and dysglycemia in CD-fed $G l p 2 r^{-1-}$ mice. Hence, $G l p 2 r^{-1-}$ mice develop dysglycemia and increased hepatic lipid 

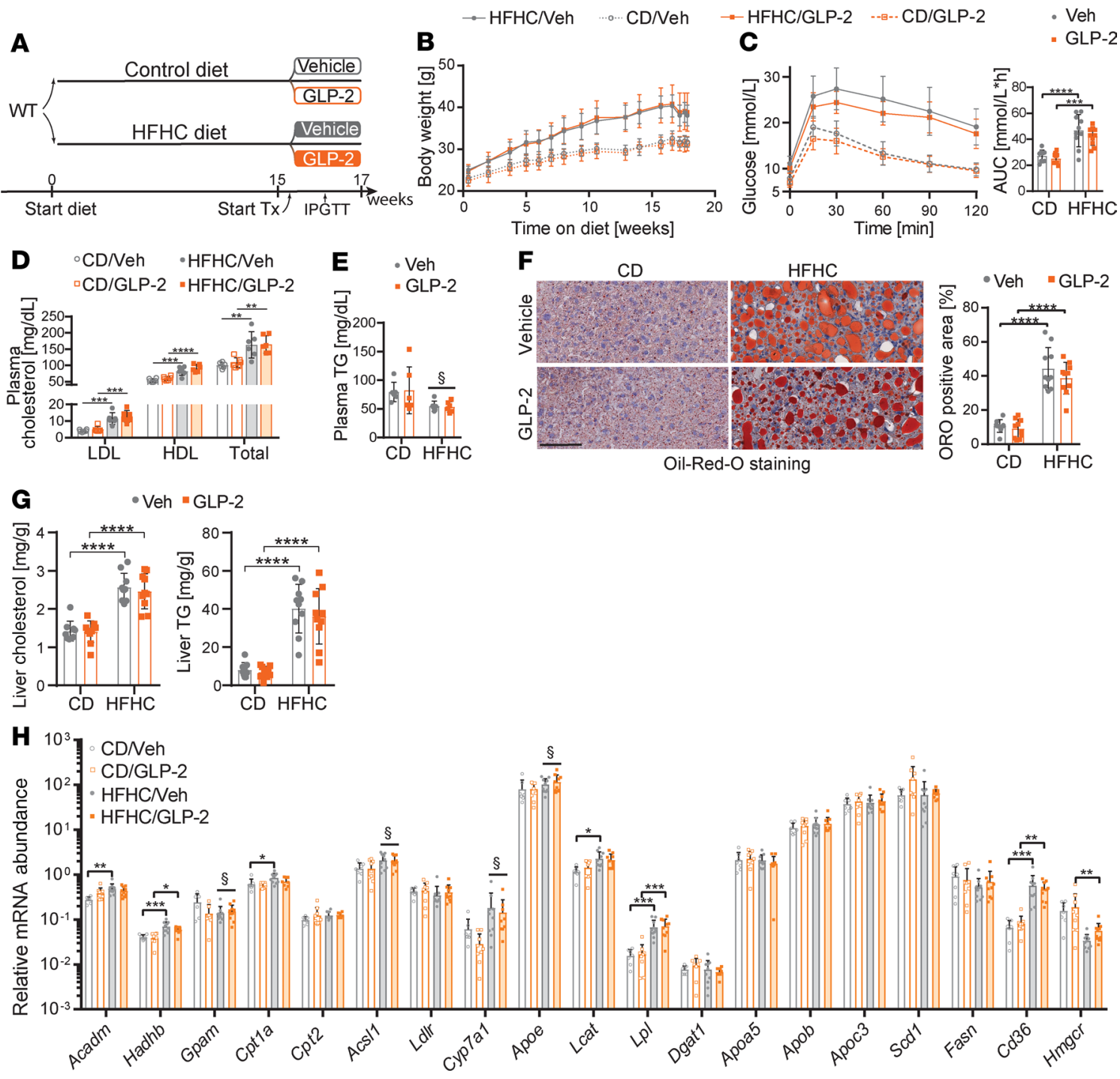

Figure 1. GLP-2 treatment does not alter glucose and lipid metabolism in HFHC diet-fed mice. Metabolic endpoints in mice with diet-induced NAFLD treated with GLP-2. (A) Seven- to eight-week-old WT male mice were allocated to HFHC or control diet (CD) for 17 weeks and then treated with h[Gly2]GLP-2 (0.1 mg/ $\mathrm{kg} \mathrm{BID)}$ or vehicle for the last 11 days on diet ( $n=9-10$ per group for all panels). (B) Body weight gain. (C) i.p. glucose tolerance in 5- to 6-hour fasted mice after 5 days of GLP-2 or vehicle and area under curve (AUC) 0-120 minutes. (D and E) Plasma lipids at the end of study. TG, triglycerides. (F) Representative Oil Red $\mathrm{O}$ staining of liver sections (scale bar: $100 \mu \mathrm{m}$ ) and quantification of Oil Red O-positive staining in liver. (G) Hepatic cholesterol and TC content. (H) Hepatic mRNA abundance, relative to Ppia, of genes relevant to lipid metabolism pathways. Data are presented as the mean \pm SD. ${ }^{*} P<0.05$, ${ }^{* *} P<0.01,{ }^{* * *} P<0.001$, ${ }^{* * * *} P<0.0001,{ }^{\circledR} P<0.05$, significant effect of diet on total variance, using 2-way ANOVA with Tukey correction for multiple comparisons.

deposition, which could not be attributed to known extrahepatic aspects of GLP-2R signaling.

Biomarkers of hepatic inflammation are selectively increased in $\mathrm{Glp}_{2 r^{-1}}$ mice. Circulating transaminase levels were markedly elevated by diet; however, ALT was further elevated in $G l p 2 r^{-1}$ mice. Aspartate transaminase (AST) levels also trended higher $(P=0.054)$ in $G l p 2 r^{-1}$ mice (Figure 4A). HFHC diet-fed mice also exhibited increased levels of hepatic cytokines. Notably, levels of TNF- $\alpha$, IL-6, and KC/GRO were higher in Glp2r/- mice (Figure 4B). Nevertheless, while plasma levels of TNF- $\alpha, \mathrm{KC} / \mathrm{GRO}$, and IL-10 were increased after HFHC feeding, plasma cytokine levels were not different in $\mathrm{Glp} 2 r^{+/+}$mice and $\mathrm{Glp} 2 \mathrm{r}^{\prime-}$ mice (Figure 4C). Similarly, HFHC diet-fed mice exhibited increased hepatic expression of several inflammatory and cellular stress genes (Figure 4D), with mRNA levels for Crp, Cxcr2, and Pkr higher in Glp2r/- mice compared with $G l p 2 r^{+/+}$mice. Interestingly, levels of Oas2, an IFN- $\gamma-$ responsive transcript, were decreased in $G l p 2 r^{\prime-}$ liver (Figure 4D).

Hence, Glp2r/- mice exhibit increased hepatic inflammation, exemplified by increases in cytokines and alterations in IFN- $\gamma$-responsive mRNA transcripts. 


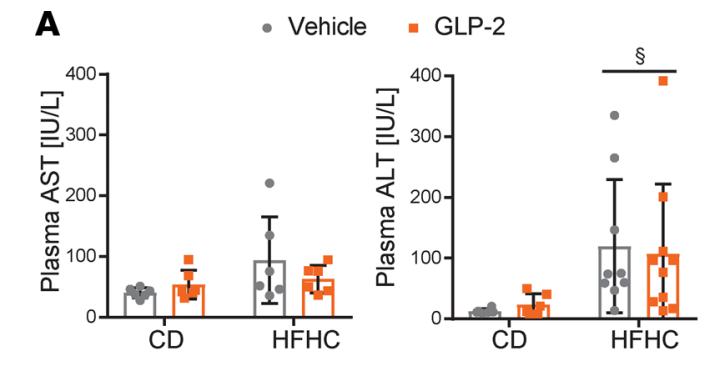

B Liver cytokines $\quad \cdot$ Vehicle = GLP-2
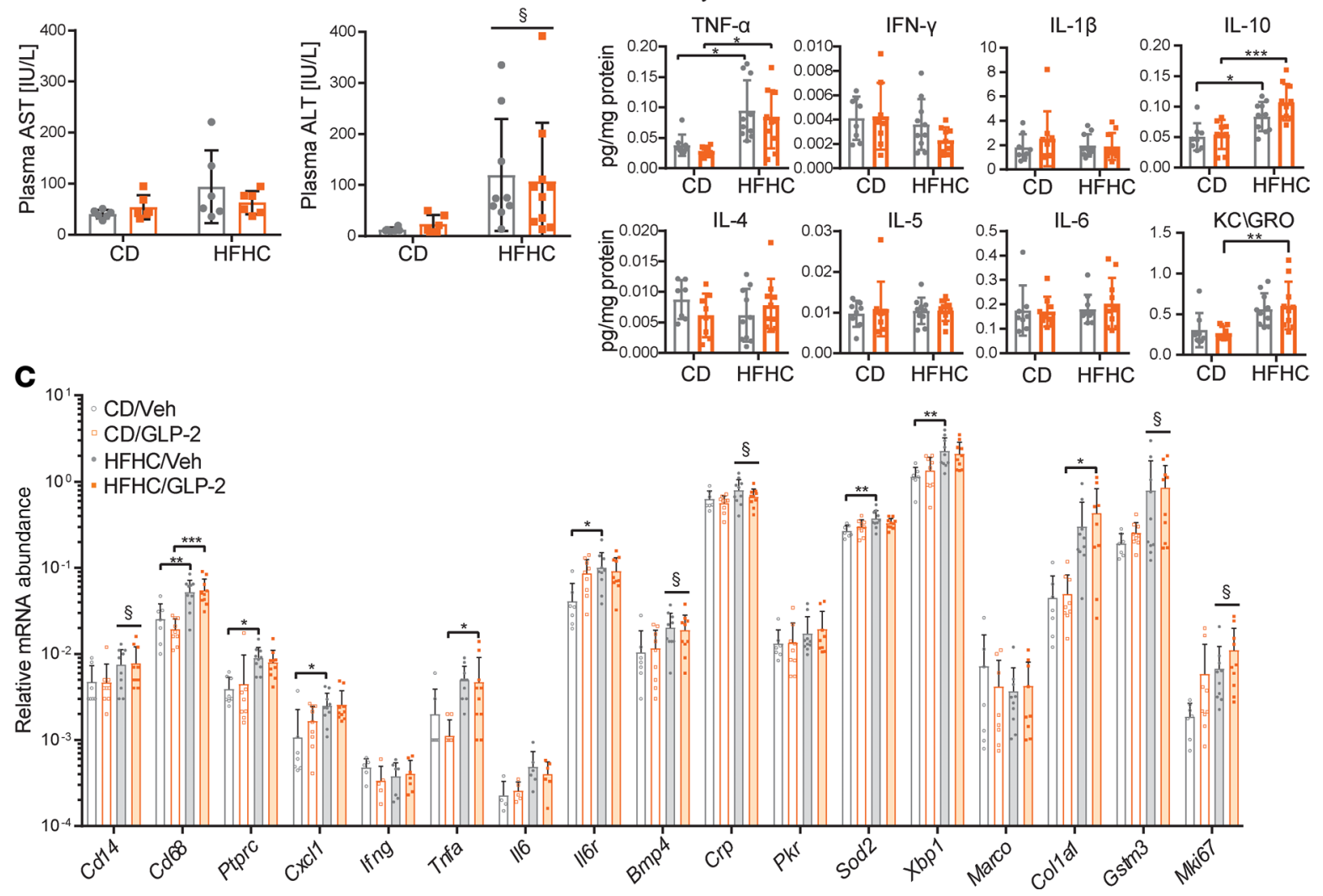

Figure 2. GLP-2 treatment in HFHC diet-fed mice does not effect hepatic inflammation. Experimental endpoints in mice fed a HFHC or CD for 17 weeks and treated with GLP-2 for 11 days, as illustrated in Figure 1A ( $n=9-10$ per group for all panels). (A) Transaminase plasma levels in cardiac blood at termination. $\S$ significant diet effect on ALT variance, $P<0.01$. (B) Protein concentrations of inflammatory markers in liver normalized to hepatic protein levels. (C) Hepatic mRNA abundance, relative to $P$ pia, of genes relevant to inflammatory pathways. Data are presented as the mean \pm SD. ${ }^{*} P<0.05,{ }^{* *} P<0.01$, ${ }^{* *} P<0.001,{ }^{\circledR} P<0.05$, significant effect of diet on gene expression variance, using 2-way ANOVA with Tukey correction for multiple comparisons.

Glp2r is expressed in HSCs. To understand how loss of the GLP-2R might predispose to increased hepatic inflammation and lipid deposition, we assessed hepatic Glp2r expression. The liver expresses low levels of mRNA transcripts corresponding to a full-length GLP-2R $(8,10)$, previously suggested to be localized to mouse and human hepatocytes (11). We detected expression of full-length GLP2R mRNA transcripts in 3 commercially available human liver total RNA samples (Figure 5A). The hepatocyte fraction isolated from mouse liver, confirmed by cell-specific marker expression (Supplemental Figure 3A), did not contain Glp2r mRNA transcripts, whereas the nonparenchymal cell (NPC) fraction contained detectable Glp2r mRNA transcripts (Figure 5B). Since HSCs are not efficiently recovered by standard NPC isolation protocols, we analyzed highly purified HSC fractions (20) (Figure 5, C-E). The purity of HSC-sorted fractions was confirmed through fluorescent microscopy (Figure 5E) and mRNA abundance of markers for HSC and nonstellate cell fractions (Figure 5D). A substantial enrichment of Glp2r expression was observed in highly purified HSCs relative to levels in total liver RNA (Figure 5F), with expression levels in HSCs approximately $50 \%$ of those detected in the jejunum. Conventional RT-PCR followed by Southern blotting identified a full-length Glp2r mRNA transcript in RNA from mouse liver and stellate cells as well as in jejunum (Figure 5G). There was no detectable expression of full-length Glp2r in RNA isolated from hepatocytes (Figure 5G and Supplemental Figure 3C).

To probe whether hepatic NPCs other than stellate cells express Glp2r, we used flow cytometry (Supplemental Figure 3B) to sort NPC subpopulations using CD26, CD31, and CD45 surface markers. $\mathrm{CD} 31^{+} \mathrm{CD} 45^{-}$(endothelial cells), $\mathrm{CD} 45^{+} \mathrm{CD} 31^{-}$(subdivided by green autofluorescence properties to Kupffer cells and other hematopoietic cells), and $\mathrm{CD} 31^{-} \mathrm{CD} 45^{-} \mathrm{CD} 26^{+}$(comprising cholangiocytes and other nonendothelial, nonhematopoietic NPCs, with the exception of HSCs that are CD26-) were further identified by 
A
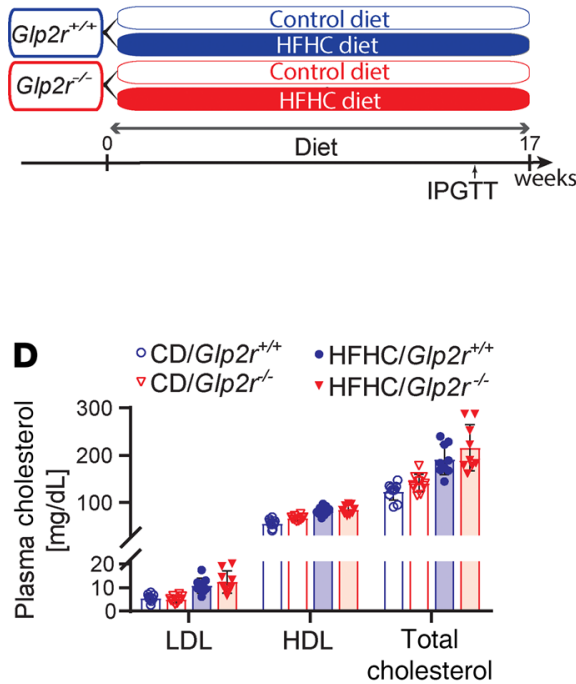

$\mathbf{F}$

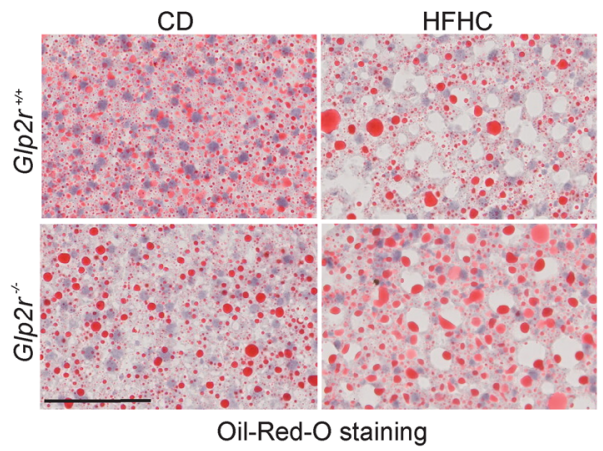

B

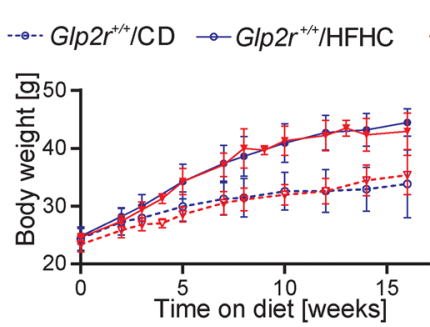

C

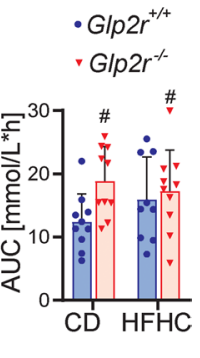

E

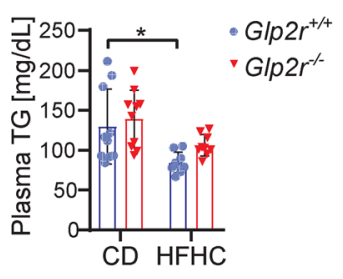

G
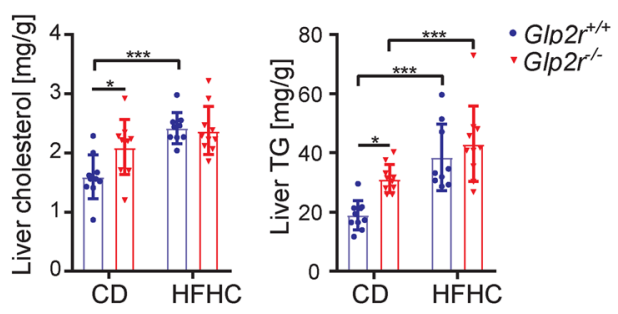

H

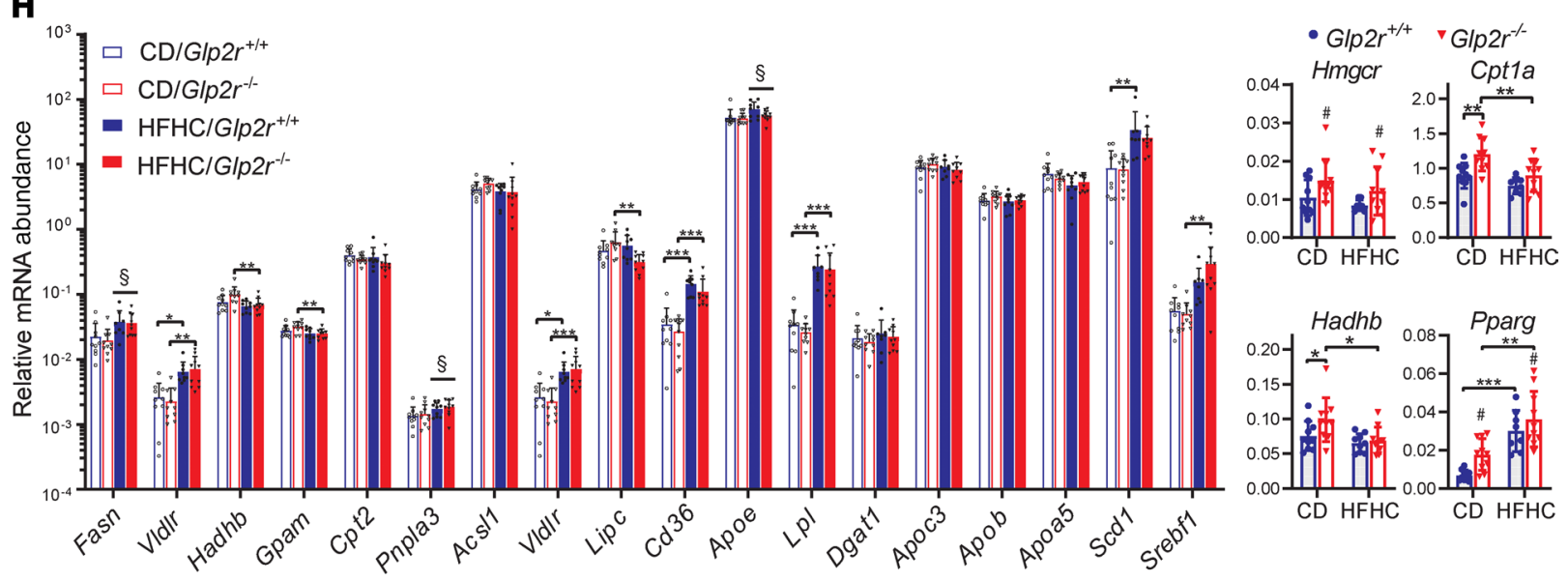

Figure 3. Glp2 $r^{-1-}$ mice exhibit dysglycemia and increased hepatic lipid deposition. Metabolic endpoints in GIp2 $r^{1++}$ and GIp2 $r^{1-}$ mice fed either a control diet (CD) or HFHC diet. (A) Seven- to eight-week-old Glp2r+/+ and Glp2r-l-male mice were allocated to CD or a HFHC diet for 17 weeks ( $n=9-10 / g r o u p$ for all panels). (B) Body weight gain. (C) i.p. glucose tolerance in 5-hour fasted mice and area under curve (AUC) 60-120 minutes. Data are presented as the mean \pm SD. ${ }^{~} P<0.05$, significant effect of genotype on AUC variance, using 2 -way ANOVA with Tukey correction for multiple comparisons. (D and E) Plasma lipid profiles at endpoint. (F) Representative Oil Red 0 staining of liver sections. Scale bar: $100 \mu \mathrm{m}$. (G) Hepatic cholesterol and triglyceride (TG) content. (H) Hepatic mRNA abundance, relative to Ppia, of genes affecting lipid metabolism pathways. Graphs on the right illustrate relative mRNA levels of $H m g c r$, Pparg, Cpt1a, and Hadhb genes, for which genotype had a significant effect on gene expression variability. Data are presented as the mean \pm SD. ${ }^{*} P<0.05$, ${ }^{* *} P<0.01,{ }^{* * *} P<0.001,{ }^{\circledR} P<0.05$ significant effect of diet, ${ }^{*} P<0.05$, significant effect of genotype on variance of gene expression, using 2 -way ANOVA with Tukey correction for multiple comparisons. 


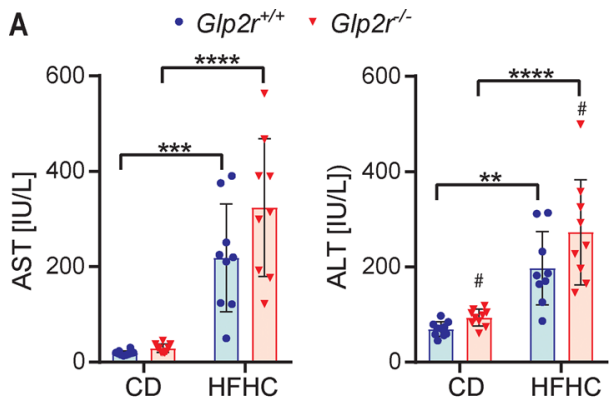

B Liver cytokines

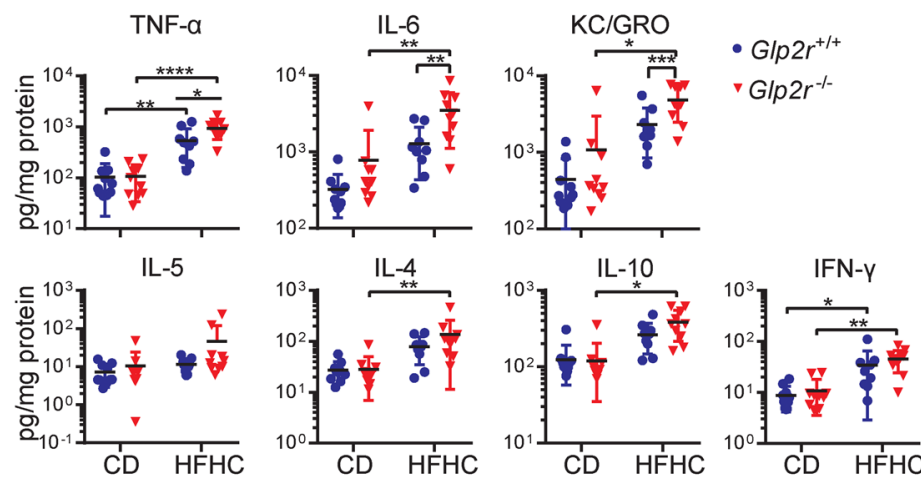

C Plasma cytokines $\quad$ Glp2r $r^{+/+} \quad$ G $/ p 2 r^{-/-}$


Figure 4. Glp2 $r^{1-}$ mice exhibit enhanced hepatic inflammation on a HFHC diet. Hepatic inflammation and fibrosis endpoints in Glp2 $r^{+/+}$or Glp2 $r^{1-}$ mice fed HFHC or CD for 17 weeks, as shown in Figure 3A ( $n=9-10 /$ group for all panels). (A) Plasma transaminase levels from cardiac blood at termination. (B) Protein levels of inflammatory markers in liver normalized to hepatic protein levels. (C) Protein concentrations of inflammatory markers in plasma. (D) Hepatic mRNA abundance, relative to Ppia, of genes relevant to inflammatory pathways. Graphs on the right illustrate relative mRNA levels of Oas2, Pkr Cxcr2, and Crp genes, for which genotype had a significant effect on gene expression variability. Data are presented as the mean \pm SD. ${ }^{*} P<0.05,{ }^{* *} P<$ $0.01,{ }^{* *} P<0.001,{ }^{* * *} P<0.001,{ }^{*} P<0.05$ significant effect of genotype on variance of gene expression, using 2 -way ANOVA with Tukey correction for multiple comparisons.

marker expression. No Glp2r was detected in any of these NPC fractions (Supplemental Figure 3, B and C).

The HSC GLP-2R is directly activated by GLP-2 in vitro. Activation of GLP-2R signaling rapidly induces immediate-early gene (IEG) expression in the intestine as well as in heterologous cells expressing the GLP2R (21). Exposure of isolated murine HSCs to GLP-2 increased levels of Egr1, Nr4a, Rasd1, and Phlda1, consistent with a functional HSC GLP-2R (Figure 5H and Supplemental Figure 4A).

Regulation of hepatic Glp2r expression. We next analyzed hepatic Glp2r mRNA expression from different groups of mice exposed to various diets. Mice fed a HFHC diet for 17 weeks exhibited an approximately 2-fold increase in hepatic Glp2r expression (Supplemental Figure 4B). A similar increase in hepatic Glp2r mRNA transcripts was detected in mice after 12 weeks of 45\% HFD feeding (Supplemental Figure 4B) as well as in mice on a methionine-choline-deficient (MCD) diet. Notably, mRNA levels of the stellate cell marker Pdgfrb, a reflection of HSC abundance, were not different (Supplemental Figure 4B).

Genotype-dependent differences in HSC mRNA transcripts. We next analyzed levels of mRNA transcripts for 11 genes reported to be upregulated in the context of HSC activation (22-25) in livers from mice fed a MCD diet for 2 weeks. Three of these markers, Timp1, Mmp13, and Adora2b, were upregulated in whole 
A
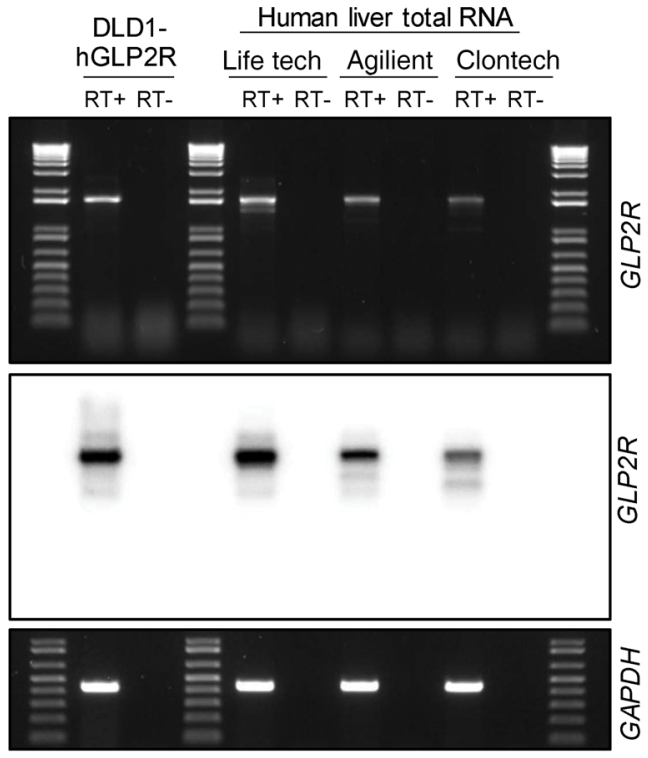

E

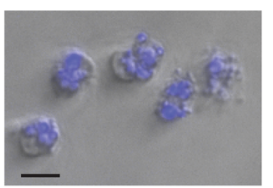

$\mathbf{F}$

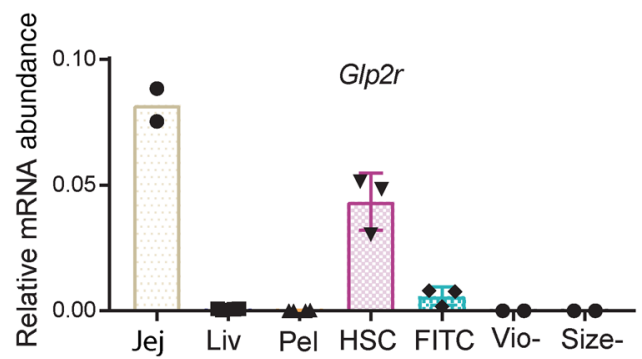

C

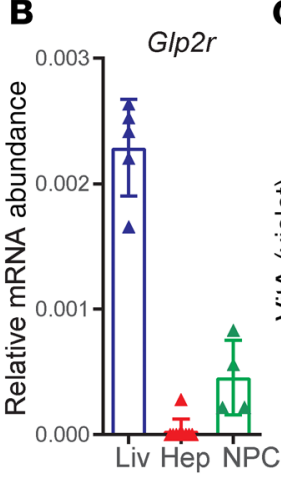

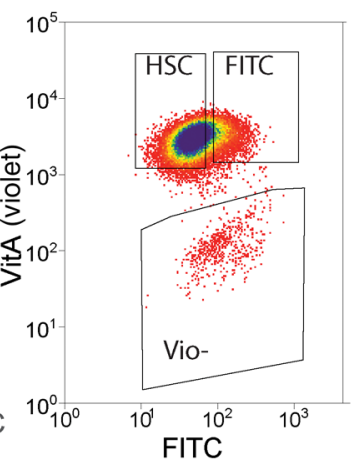

D

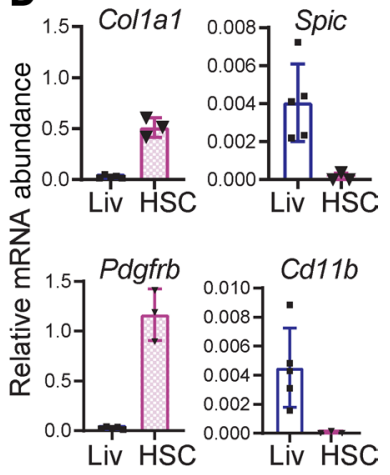

G
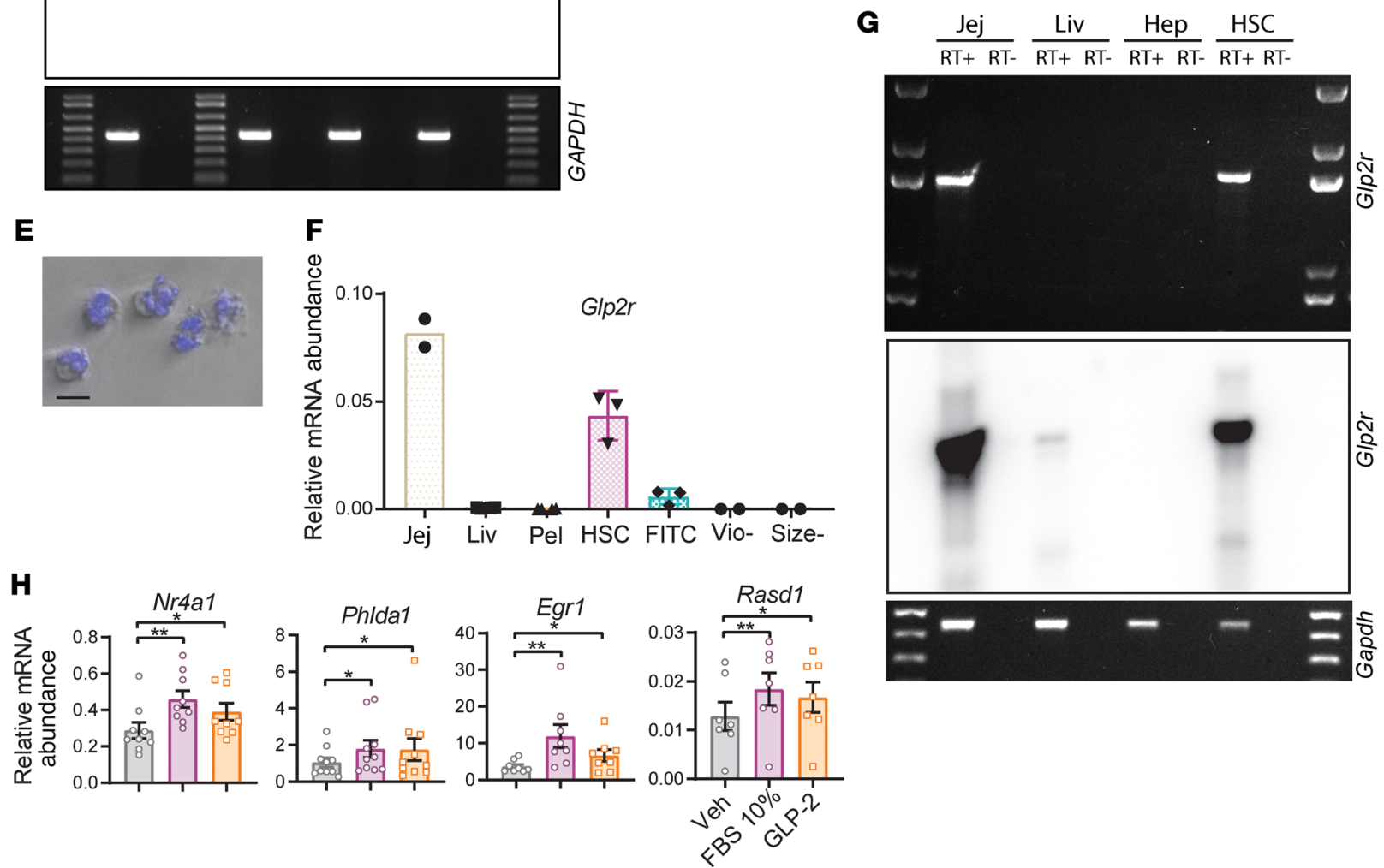

Figure 5. The canonical GIp2r is expressed in hepatic stellate cells. (A) The expression of human GLP2R and GAPDH transcripts was assessed by RT-PCR in total RNA in human livers obtained from 3 indicated commercial sources and in RNA from a human GLP2R expressing human colon cancer epithelial cell line (DLD-1) as a positive control. For reverse transcription PCR of GLP2R we used primers that amplify a 1.687-kb product encompassing the entire GLP2R open reading frame. PCR products were analyzed by agarose gel electrophoresis followed by SYBR green staining (top and bottom) or by Southern blot with an internal GLP2R ${ }^{32}$-P-labeled oligonucleotide (middle). RT, reverse transcriptase. (B) Levels of Glp2r mRNA, relative to Ppia, in whole liver (Liv, $n=6$ ), isolated hepatocytes (Hep, $n=10$ ), and nonparenchymal liver cells (NPC, $n=4$ ). (C) Hepatic stellate cells (HSC; $n=3$ ) purified by centrifugation and flow cytometry. (D) mRNA abundance, relative to Ppia, of HSC (Col1a1, Pdgfrb) and nonstellate cell (Spic, Cd11b) markers in purified HSCs and whole liver. (E) Microscopy image demonstrating lipid vacuole autofluorescence in purified HSC. Scale bar: $20 \mu \mathrm{m}$. (F) Glp2r mRNA abundance, relative to Ppia, in jejunum (Jej), liver (Liv), pellet collected after Nycodenz gradient isolation of HSC (Pel), purified HSC fraction gated by size and positive violet autofluorescence (HSC), cells positive for violet and FITC autofluorescence that correspond with HSCs mixed with contaminant cells (FITC), cells gated negative to violet autofluorescence (Vio-), and cells outside size gate (Size-). (G) Full-length mouse GIp2r expression in jejunum, whole liver, hepatocytes, and isolated HSCs. Glp2r mRNA transcripts were amplified by reverse transcription PCR with primers that amplify a 1.65-kb product encompassing the entire GIp2r open reading frame. PCR products were analyzed by agarose gel electrophoresis followed by SYBR green staining (top and bottom). Southern blotting with an internal Glp2r ${ }^{32}$-P-labeled oligonucleotide (middle) was used to detect the PCR product. (H) mRNA abundance, relative to Ppia, of immediate-early genes used as markers of GLP-2 action in isolated HSCs. Hepatic stellate cells were purified from 18 C57BL/6 WT mice at 4-6 months old. Cells, combined from 1-4 mice per data point, were incubated for 20 minutes with either $10 \%$ FBS, GLP-2 (50 nM), or saline (Veh). Data are presented as the mean \pm SEM. ${ }^{*} P<0.05,{ }^{*} P<0.01$, using ratio-paired $t$ test. 
liver (Supplemental Figure 4C). In a repository of data from hepatocyte single-cell RNA sequencing (12), these 3 markers were expressed at negligible levels in hepatocytes. Thus, we infer that stellate cell activation markers can be informative from analysis of whole liver RNA. Consistent with these findings, we detected increased transcript abundance for stellate cell markers Pdgfrb, Mmp13, Timp1, and Col8a1 after HFHC feeding, with higher levels for Timp1 and Colsa1 detected in livers from Glp2r-1- mice compared with those from $G_{p l p} 2 r^{+/}$mice (Figure 6A). However, liver fibrosis, quantified by Sirius red staining (Figure 6B), was increased in HFHC-fed mice but not different in $G l p 2 r^{+/+}$mice compared with $G l p 2 r^{-1-}$ mice, and mRNA levels of the fibrogenic markers Colla1 and Acta2 were comparable in Glp2r+/+ mice and Glp2r/- mice (Figure 6B). Furthermore, levels of mRNA transcripts for Colla1 and Acta2, encoding markers of fibrosis, were also increased in hepatic RNA from 50-week-old Glp2r/- mice (Figure 6C).

Activation or loss of GLP-2R signaling does not effect the hepatic response to $\mathrm{CCl}_{4}$. To assess the importance of the GLP-2R in an independent model of hepatic injury, we used the profibrotic agent $\mathrm{CCl}_{4}$. WT male C57/BL6 mice were treated with i.p. $\mathrm{CCl}_{4}$ every 3 days, for a total of 4 doses (26). Starting 2 days prior to initiation of $\mathrm{CCl}_{4}$ treatment and until termination 3 days after the last $\mathrm{CCl}_{4}$ injection, mice were treated daily with GLP-2 or vehicle (Figure 7A). $\mathrm{CCl}_{4}$-treated mice had elevated levels of plasma ALT, increased liver fibrosis (as approximated by Sirius red staining), and elevated levels of mRNA transcripts for several HSC activation markers analyzed in whole liver samples and in purified HSC preparations (Figure 7, B-E). Nevertheless, GLP-2 treatment had no effect on any of these endpoints or on body weight (Figure 7, B-E, and Supplemental Figure 4D). Unexpectedly, HSC Glp2r expression was approximately 8-fold lower in $\mathrm{CCl}_{4}$-treated mice (Figure 7E). We next studied the consequences of $\mathrm{CCl}_{4}$ administration in $\mathrm{Glp}^{2 r^{+/+}}$and Glp2 $r^{-1}$ male mice (Figure 8A). Plasma ALT, the extent of Sirius red staining, and mRNA levels of HSC activation markers in the whole liver were generally similar between groups (Figure 8, B-E). Interestingly, mRNA levels of Lox, frequently induced in early stages of HSC activation (27), were upregulated in HSCs from Glp2r/- mice (Figure 8E).

\section{Discussion}

The current studies were prompted in part by questions surrounding the use of the GLP-2 analog teduglutide in human subjects with intestinal failure at risk for development of $\mathrm{PN}$-associated hepatosteatosis (28) and the conflicting data surrounding the putative actions of GLP-2 in the liver (13, 17, 29). GLP-2 rapidly increases postprandial levels of TGs in mice, hamsters, and humans (30-32), suggesting that increased substrate availability may contribute to hepatosteatosis in the setting of sustained GLP-2R signaling. Indeed, Taher and colleagues demonstrated increased hepatic lipogenesis in HFD-fed hamsters treated with GLP-2 as well as in rodent chow-fed (RC-fed) mice treated with GLP-2 (13). In contrast, treatment of HFDfed mice with the GLP-2R antagonist GLP-2(3-33) exacerbated hepatic steatosis in HFD-fed mice (17), whereas GLP-2 administration attenuated development of hepatosteatosis in PN-fed rats after small bowel resection (29). Our data, using administration of a degradation-resistant GLP-2R agonist, revealed no compelling effect of GLP-2R activation on hepatic steatosis in WT HFHC-fed mice. Collectively, our data are consistent with findings that teduglutide administration produced no change or reduced levels of transaminases and bilirubin in humans with SBS (33).

In contrast, we demonstrated that genetic loss of the Glp2r increased the extent of hepatic steatosis and upregulated hepatic cytokine expression in mice. These findings are consistent with data from Baldassano (17) and Cani (14), who demonstrated a protective role for GLP-2R signaling in HFD-fed mice and $o b /$ $o b$ mice treated with or without prebiotics and GLP-2 or a GLP-2R antagonist, respectively. Intriguingly, recent evidence links HSC activity to de novo lipogenesis and the development of experimental steatosis (34). Hence, our current findings of hepatosteatosis and increased hepatic inflammation in $G l p 2 r^{1-}$ mice are consistent with a putative mechanism arising from loss of the HSC GLP-2R or potential consequences of absent GLP-2R signaling affecting control of gut barrier function, inflammation, and hepatic insulin sensitivity (14). The precise mechanisms linking loss of GLP-2R activity to increased hepatic fat accumulation remain uncertain and require additional investigation.

Despite multiple studies examining the roles of GLP-2R in liver biology, the precise cellular localization of the hepatic GLP-2R has remained uncertain. We localized the hepatic GLP-2R to stellate cells and not, as previously reported (11), in hepatocytes. Intriguingly, GLP-2 infusion rapidly enhances the appearance of circulating TG-rich lipoprotein-associated retinyl palmitate (35), establishing a putative link among GLP-2 action, vitamin A bioavailability, and subsequent storage in HSCs. Indeed, the HSC GLP- 
A

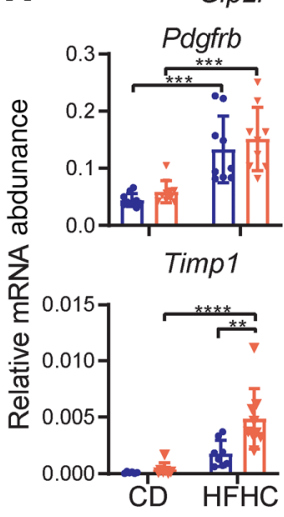

- $\mathrm{Glp} 2 \mathrm{r}^{+/+} \nabla \mathrm{Glp} 2 \mathrm{r}^{-/-}$

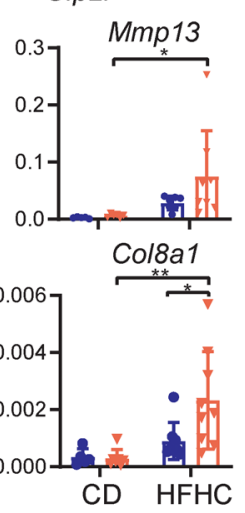

B

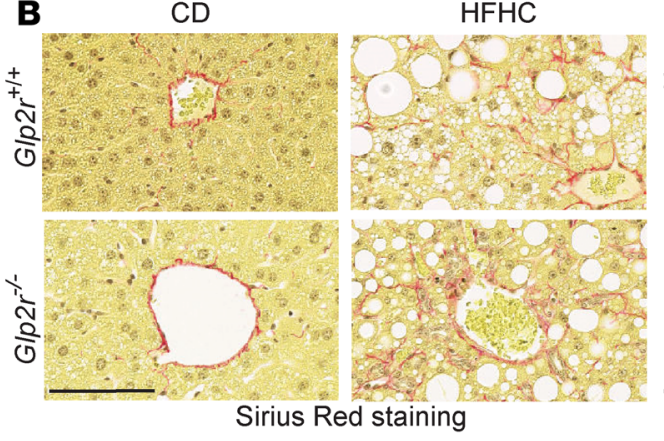

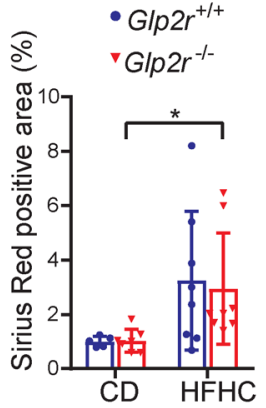

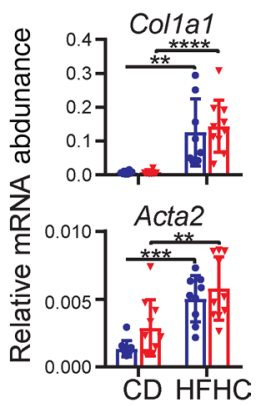

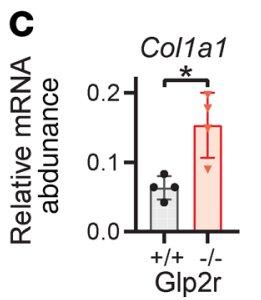
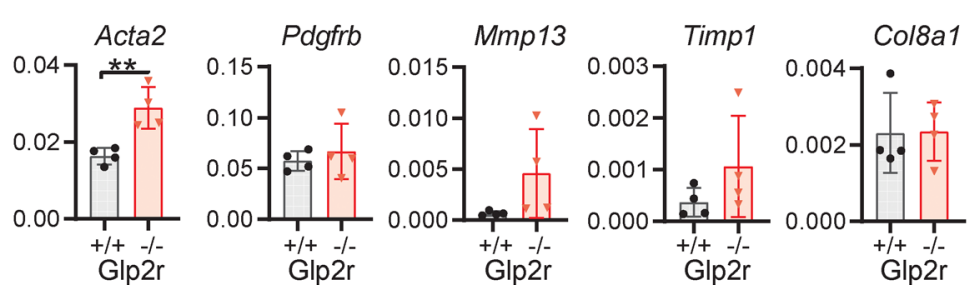

Figure 6. Hepatic stellate cell activation markers are elevated in Glp2 $\mathbf{r}^{-1-}$ mice on HFHC diet and in aged mice. (A) mRNA abundance, relative to Ppia, of hepatic stellate cell activation markers in hepatic RNA from GIp2 $r^{+/+}$and Glp2 $r^{-1-}$ mice on HFHC or CD as outlined in Figure $3 A$ ( $n=9-10$ per group). (B) Representative Sirius red staining of liver sections with quantification of Sirius red-positive staining (left) and liver mRNA abundance, relative to Ppia, of fibrosis markers Col1a1and Acta2 (right) ( $n=9-10$ per group). Scale bar: $100 \mu \mathrm{m}$. (C) mRNA levels, relative to Ppia, of stellate cell activation markers in livers from GIp $2 r^{+/+}$and GIp $2 r^{-1-}$ male mice age $50 \pm 3.5$ weeks old $\left(n=4\right.$ each). Data are presented as the mean $\pm \mathrm{SD}$. ${ }^{*} P<0.05$, ${ }^{* *} P<0.01,{ }^{* * *} P<0.001,{ }^{* * * *} P<$ 0.0001 , using 2-way ANOVA with Tukey correction for multiple comparisons or Student's $t$ test where appropriate.

2R was functional, as GLP-2 directly induced a subset of IEGs in stellate cells ex vivo, findings consistent with previous studies of GLP-2R signaling ex vivo (36). Notably, relative Glp2r expression in purified HSCs approximates levels detected in RNA isolated from the jejunum, a major site of GLP-2R expression (37). Our data are also consistent with the detection of HSC Glp2r expression in studies analyzing single-cell RNA-sequencing data from murine stellate cells (38).

Interestingly, HSC Glp2r transcripts were upregulated after exposure to various diets used for experimental induction of hepatic injury, including a 45\% HFD, a HFHC diet, and a MCD diet. These 3 different diets promote hepatic steatosis; however, the MCD diet induces a milder degree of steatosis, no weight gain, and increased hepatic inflammation and fibrosis (39). Conversely, mice with $\mathrm{CCl}_{4}$-induced liver injury exhibited a marked reduction in Glp2r expression within stellate cells. Collectively, these data show for the first time to our knowledge that Glp2r expression is dynamically regulated in the injured liver and dietary versus chemical hepatic insults produce divergent regulation of HSC Glp2r expression. These observations reveal the importance of interpreting hepatic GLP-2 action in the context of potential changes in expression of the endogenous GLP-2R.

Current concepts linking the gut to stellate cell activation highlight roles for microbial metabolites, including bile acids (40), as well as bacterial cell wall products, exemplified by lipopolysaccharide (41), which engage HSCs via distinct receptors and signaling pathways. Notably, bacterial metabolites (42) and cell membrane constituents such as LPS $(43,44)$ as well as bile acids $(45)$ are also potent stimulators of GLP-1 and GLP-2 secretion from enteroendocrine L cells (46). Our current findings demonstrate that loss of basal GLP-2R signaling is associated with HSC activation in mice with diet-induced hepatic injury. Collectively, these observations expand the concept of a gut-HSC axis through identification of a functional GLP-2R signaling system within HSCs, diversifying the mechanisms through which nutritional stimuli and microbiota may indirectly modulate HSC activity.

Our studies have several limitations. First, we used whole body $G l p 2 r^{-1}$ mice with germline loss of the Glp2r that may exhibit developmental or functional compensation, such as elevated levels of GLP1 as shown here. Tissue-specific and conditional inactivation of the Glp2r selectively within HSCs 
A

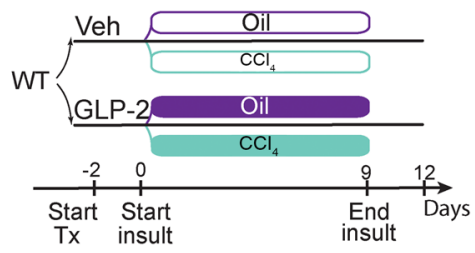

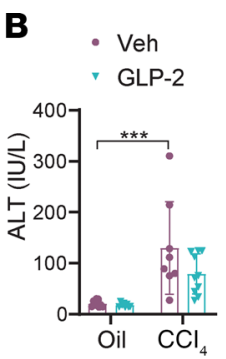

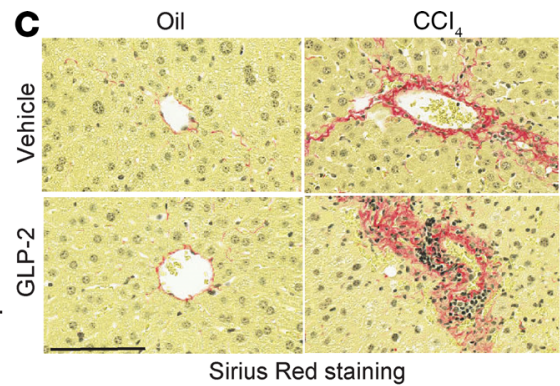

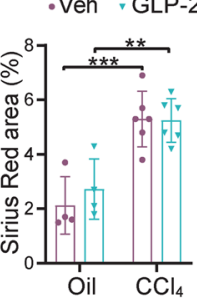

D Whole liver
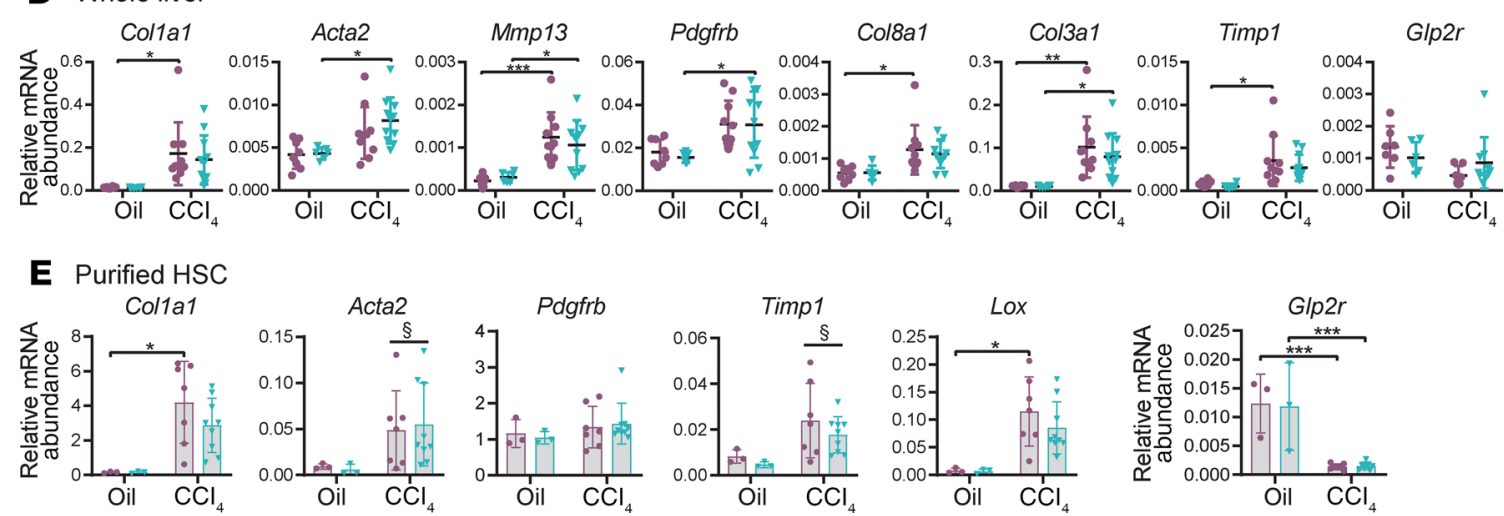

Figure 7. GLP-2 treatment does not change $\mathrm{CCl}_{4}$-mediated liver injury and fibrosis. Fibrosis, liver enzymes, and RNA levels of genes reflecting hepatic stellate cell (HSC) activation in $\mathrm{C} 57 \mathrm{BL} / 6$ mice treated with $\mathrm{CCl}_{4}$ or mineral oil vehicle, together with daily $\mathrm{CLP}-2$ (0.2 mg/kg, SC) or vehicle. (A) Mice were pretreated with once-daily GLP-2 or vehicle starting 2 days prior to initiation of $\mathrm{CCl}_{4}$ treatment. A total of $4 \mathrm{CCl}_{4}(0.7 \mathrm{ml} / \mathrm{kg})$ or vehicle i.p. injections were administered (once every 3 days), and then mice were sacrificed 3 days after the last $\mathrm{CCl}_{4}$ injection. GLP- 2 was continued until the final day ( $n=8-11$ per group). (B) Plasma ALT levels at sacrifice. (C) Representative Sirius red staining of liver sections (scale bar: $100 \mu \mathrm{m}$ ) and quantification of Sirius red-positive area. (D) mRNA abundance of genes corresponding to markers of fibrosis and stellate cell activation in whole liver, normalized to Ppia. (E) mRNA abundance of reporters in purified hepatic stellate cells (HSC), normalized to Pdgfrb. Each data point represents cells combined from 1-4 mice. Data are presented as the mean $\pm \mathrm{SD}$. ${ }^{*} P<0.05,{ }^{* *} P<0.01,{ }^{* *} P<0.001,{ }^{\circledR} P<0.05$ significant effect of treatment on gene expression variance, using 2 -way ANOVA with Tukey correction for multiple comparisons.

would further establish the role of the HSC GLP-2R in the phenotypes observed in our studies. Moreover, we studied predominantly younger mice, and stellate cell activation is known to become more prominent with increasing age (47). Additionally, manipulation of central nervous system GLP-2R signaling has been shown to modulate hepatic insulin sensitivity (48), a pathway that was not directly examined in the current studies. In addition, we were unable to detect GLP-2R protein within HSCs due to limitations in the sensitivity and specificity of available GLP-2R antisera (7). Nevertheless, our experiments establish the presence of a full-length Glp2r mRNA and functional GLP-2R in HSCs, findings with potential importance for understanding how gut-derived signals effect HSC biology in a broader context.

Given the use of teduglutide in human children and adults with SBS and hepatosteatosis, taken together with the emerging importance of HSC activation in the pathophysiology of nonalcoholic fatty liver disease, our findings have translational relevance for understanding how the gut, microbiota, and microbial metabolites modify stellate cell adaptive responses to nutritional stress, inflammation, and hepatic injury.

\section{Methods}

\section{Animal care and genotyping}

Mice were housed (2-5 mice per cage), under a 12-hour-light/dark cycle in the Toronto Centre for Phenogenomics (TCP). Experiments were started in mice at 7-9 weeks of age or older if involving stellate cell isolation, as indicated in the figure legends. Age-matched mice were randomly assigned to experimental groups. All experiments were done in male mice.

WT C57B1/6 male mice were purchased from The Jackson Laboratory, and Glp2r/- mice, generated in-house (49) on a C57Bl/ 6 background, were bred at the TCP animal facility. Genotyping was performed 
A

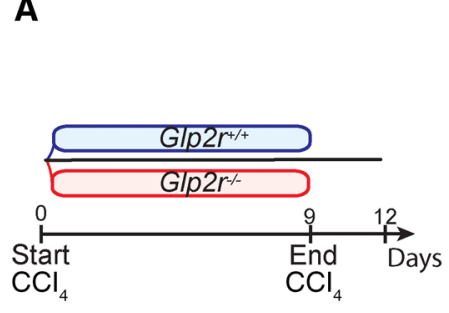

B

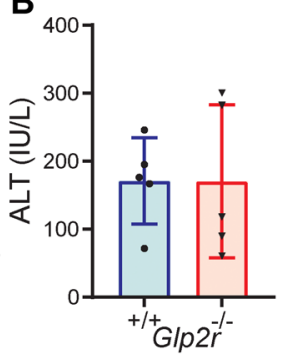

C Sirius Red staining
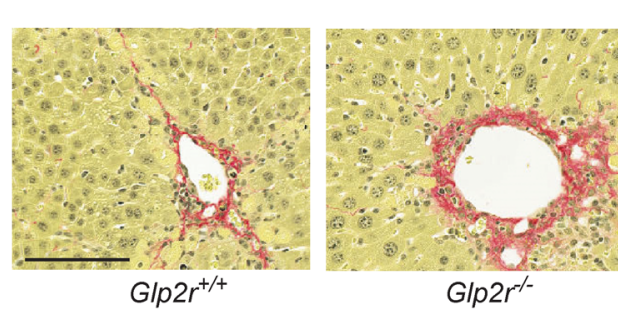

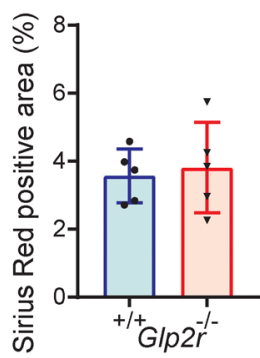

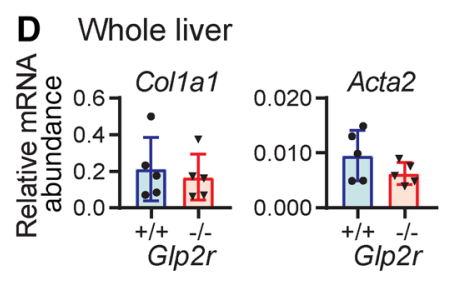
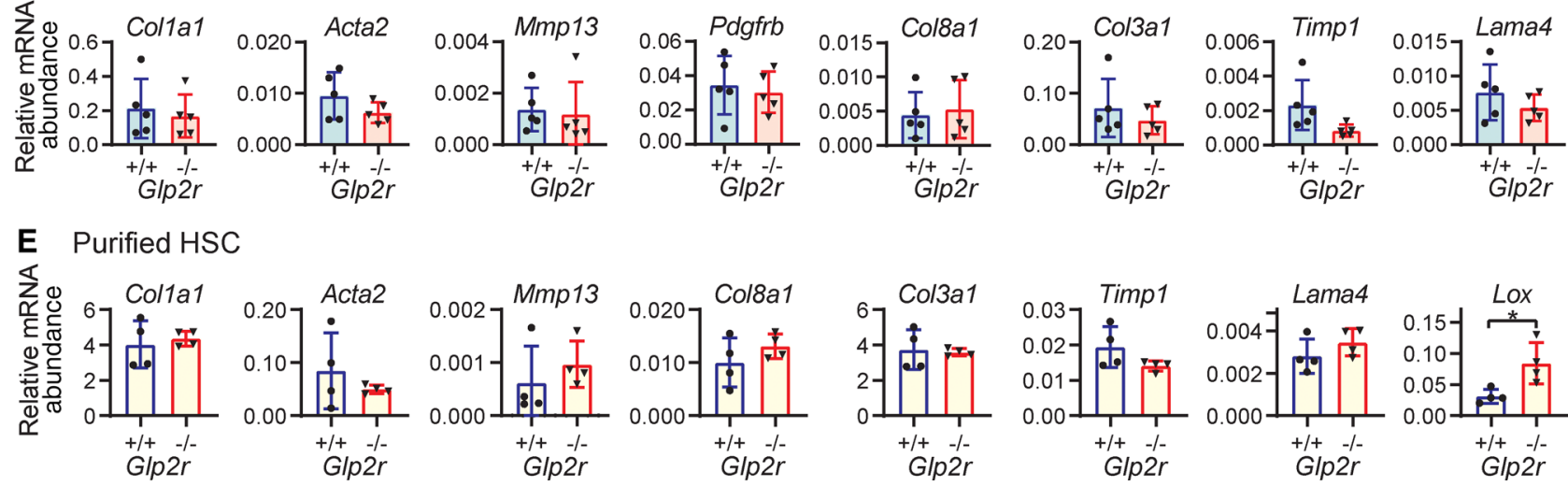

Glp2r

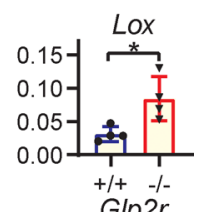

Figure 8. Loss of GLP2R function does not effect $\mathrm{CCl}_{4}$-mediated liver injury and fibrosis. $\mathrm{Glp} 2 \mathrm{r}^{+/+}$and $\mathrm{Glp} 2 \mathrm{r}^{1-}$ mice were treated with a total of $4 \mathrm{CCl}_{4}(0.7$ $\mathrm{ml} / \mathrm{kg}$ ) i.p. injections (once every 3 days), and then sacrificed 3 days after the last $\mathrm{CCl}_{4}$ injection ( $n=5 /$ group). (A) Experimental design. (B) Plasma ALT lev-

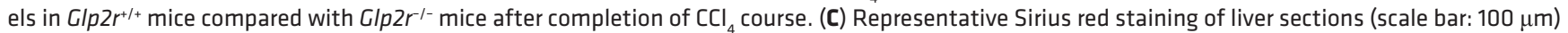
and quantification of Sirius red-positive area. Relative mRNA levels of markers of fibrosis and stellate cell activation after completion of $\mathrm{CCl}_{4}$ administration in RNA from (D) whole liver normalized to Ppia, and (E) purified hepatic stellate cells (HSC) normalized to Pdgfrb. Data are presented as the mean \pm SD. ${ }^{*} P<0.05$, using Student's $t$ test.

by PCR on tail DNA as previously described (49).

\section{Mouse diets}

All mice were fed a standard RC diet (18\% kcal from fat, 2018 Harlan Teklad) until initiation of experimental diet at age 7-9 weeks. The HFHC arm was introduced as follows. Mice were started on 2 weeks of $45 \%$ HFD (45\% kcal from fat, Research Diets, D12451i) and then switched to 3 weeks of $60 \%$ HFD $(60 \% \mathrm{kcal}$ fat, Research Diets, D12492), followed by 12 weeks of a HFHC diet (40\% kcal fat, $20 \% \mathrm{kcal}$ fructose, and $2 \%$ cholesterol, Research Diets, D09100301). The appropriate matching CD (10\% $=$ kcal fat, $0 \%$ fructose, and $0 \%$ cholesterol, Research Diets, D09100304) was administered in parallel to HFHC feeding to control mice.

Rationale for choice of diet. Fructose-containing diets promote hepatic de novo lipogenesis and fat oxidation and modify the effects of a HFD on the development of insulin resistance (50). They are also associated with higher degrees of hepatic inflammation and fibrosis (51).

Additional cohorts. For data shown in Supplemental Figure 4, B and C, 12-week-old WT C57BL/6 male mice were maintained on a MCD or methionine-choline-sufficient diet for 14 days (Cedarlane, A02082002Bi or A02082003Bi, respectively). For data shown in Supplemental Figure 4B, 8-week-old WT mice were fed RC or 45\% HFD (Research Diets, D12451i) for 12 weeks. For data shown in Figure 6C, age-matched $\mathrm{Glp}_{2 r^{+/+}}$and $\mathrm{Glp} 2 r^{-/}$mice were bred and housed in the TCP animal facility and fed a regular chow diet until age 1 year.

Excluded mice. One mouse from the experiment described in Figures 1 and 2 on $\mathrm{CD}$ was excluded prior to allocation to GLP-2 treatment due to unexplained extremely poor weight gain. One mouse from the experiment described in Figures 3 and 4 from the $\mathrm{Glp}_{2 r^{+/+}}$group fed HFHC was excluded from analyses after repeat validation genotyping after sacrificing revealed $G l p 2 r$ heterozygosity. 


\section{GLP-2 treatment}

Mice were injected subcutaneously with $0.2 \mathrm{mg} / \mathrm{Kg} / \mathrm{d}$ of the long-acting GLP-2 analog h[Gly2]GLP-2. Custom synthesized human [Gly2]GLP-2, used for the experiment described in Figures 1 and 2, was from Pepceuticals Ltd. Human [Gly2]GLP-2 (Teduglutide), used for the experiment described in Figure 7 and Supplemental Figure 4D, was a gift from Shire-Takeda Inc.

GLP-2 was administered twice daily at 9 am and 5 pm for 11 days (for the experiment described in Figures 1 and 2) or once daily at 9-10 am for 14 days (for the experiment described in Figure 7). Control mice were treated with saline in the same volume. GLP-2 doses and length of treatment were based on previous studies describing the intestinotrophic effects.

\section{CCI4-induced liver fibrosis model}

Liver injury was induced by i.p. injections of $0.7 \mu \mathrm{l} / \mathrm{g} \mathrm{CCl}_{4}$ (Toronto Research Chemicals, C176905), dissolved in mineral oil (MilliporeSigma) (52). Injections were administered in 3-day intervals for a total of 4 injections as described in ref. 20. Euthanasia took place 3 days after the last injection.

\section{Blood and tissue collection}

Blood was taken via the tail vein during metabolic studies. For isolation of liver fractions, mice were anesthetized with Avertin. For other terminal studies, mice were sacrificed by $\mathrm{CO}_{2}$ inhalation, and blood was collected via cardiac puncture into $1-\mathrm{ml}$ syringes washed with heparin $(10$ units $/ \mathrm{ml})$ and centrifuged at $14,000 \mathrm{~g}$ for 10 minutes at $4^{\circ} \mathrm{C}$ to collect the plasma. Plasma samples were stored at $-80^{\circ} \mathrm{C}$ until further analyses.

Intestines were flushed with PBS and omental fat was removed prior to weight and length measurements. Sections were obtained from the proximal jejunum for RNA and protein analyses. Gallbladder volume measurement was performed as described previously (8). Briefly, the gallbladder was ligated to prevent emptying and resected prior to liver manipulation. A photograph of the gallbladder was then taken next to a ruler, and volume was calculated as ellipsoid volume, assuming both short diameters to be equal. The liver was resected and weighed after removal of gallbladder.

\section{Glucose tolerance test}

After a 5- to 6-hour fast, glucose (1.5 mg/g body wt; MilliporeSigma) was administered through i.p. injection. A blood sample was drawn from a tail vein at 0, 10, 20, 30, 60, 90, and 120 minutes after glucose administration, and blood glucose levels were measured using a Glucometer Elite blood glucometer (Bayer).

\section{Lipid tolerance test}

RC diet-fed Glp2r+/+and Glp2r/- littermate mice (8-12 weeks old) were gavaged orally with $200 \mu 1$ olive oil (MilliporeSigma) following a 5- to 6-hour fast. Tail vein blood samples were collected into heparin-coated capillary tubes (a) before gavage only for DPP-4 activity; (b) before and 1, 2, and 3 hours after gavage for measurement of TG; and (c) before and 10 minutes after gavage for measurement of active GLP-1. Plasma was prepared from the collected blood samples as described above.

\section{Metabolic measurements}

Plasma. For the lipid tolerance test (Supplemental Figure 2, C-G) Plasma TG were measured using an enzymatic assay (Roche Diagnostics, 11877771216 ) and the calibrator 464-01601 (Wako). Active GLP-1 was measured in plasma using a Mesoscale assay (K150JWC-2). DPP-4 activity was measured in $10 \mu$ plasma using a fluorometric assay (substrate: 10 mM H-Gly-Pro-AMC HBr; Bachem, I-1225; standard: AMC, Bachem, Q-1025).

All other analytes in plasma were performed by the pathology core at The Centre for Phenogenomics. The Beckman Coulter AU480 clinical chemistry analyzer, which measures analytes in plasma or serum samples using photometry testing, was used in combination with appropriate reagents (ALT, AST, TG, cholesterol, LDL, and HDL), calibrators (Beckman Coulter Lyophilized Chemistry Calibrator Levels 1 and 2), and quality control materials (Bio-Rad Liquid Assayed Multiqual 1 and 3).

Liver. For determination of TG and cholesterol levels in liver, hepatic lipids were extracted following a protocol adapted from Folch et al. (53). Briefly, frozen liver samples were weighed ( $20 \mathrm{mg}$ ) and lipids were extracted using a 2:1 chloroform-methanol solution, dried under $\mathrm{N}_{2}$, reconstituted in $50 \mu 13: 2$ tert-butyl 
alcohol/Triton X-100/methyl alcohol (1:1), and quantified with commercially available enzymatic assay kits (TG kit COBAS, 7193130; Cholesterol kit Wako Diagnostics, 999-02601).

\section{Measurement of plasma and hepatic cytokine levels}

Cytokines were measured in $25 \mu \mathrm{l}$ mouse plasma or in 40-70 mg liver tissue homogenized in $500 \mu \mathrm{l}$ ice-cold lysis buffer (50 mM Tris HCl, pH 8, 1 mM EDTA, 10\% glycerol [wt/vol], 0.02\% Brij-35 [wt/vol]). Lysis buffer was supplemented with $1 \mathrm{mM}$ dithiothreitol and protease inhibitor for cytokine assay only. Tissue and plasma (containing 10\% TED) levels of cytokines were measured using the MSD V-PLEX Mouse Proinflammatory Panel assay kit (Meso Scale). Cytokine concentrations in tissue were normalized to total protein amount measured by Bradford Assay.

\section{Liver histology}

Liver samples were fixed in $10 \%$ buffered formalin for 48 hours and then transferred into $70 \%$ ethanol until processing. For Oil Red O staining, liver tissue was flash frozen in OCT compound. Samples were paraffin embedded, sectioned at 4- $\mu \mathrm{m}$ thickness, slide mounted, and stained with H\&E, Picrosirius red stain, or Oil Red O using standard protocols. Sections were scanned using the ScanScope CS System (Aperio Technologies), and digital images were captured at $\times 10$ magnification using ImageScope Software (Aperio Technologies) or an OlyVIA 2.9 system (OLYMPUS). Immunostaining was performed by the pathology core at The Centre for Phenogenomics.

Tissue fibrosis was analyzed in Sirius red-stained samples by red pixel count corresponding to stained collagen using the Aperio Imagescope Positive pixel count program that was calibrated once and applied uniformly to $5 \times 10$ images from each sample. The percentage of red pixels in total pixel area was determined. Tissue lipids were quantified in a similar manner using Oil Red O-stained sections. Three to four images per sample were analyzed using ImageJ software (NIH) reporting red and total pixel counts.

\section{RNA isolation, cDNA, and qPCR}

Tissue samples were dissected, weighed, frozen in liquid nitrogen, and stored at $-80^{\circ} \mathrm{C}$. Frozen tissues were homogenized in Tri Reagent (Molecular Research Center) using a TissueLyser II system (Qiagen), and total RNA was extracted using the manufacturer's protocol. Reverse transcription was performed with RNA treated with DNase I (Thermo Fisher Scientific, EN0521) using random hexamers (Thermo Fisher Scientific, 58875) and SuperScript III (Thermo Fisher Scientific, 18080-044). The resultant cDNA was used to assess mRNA expression by real-time quantitative PCR (QuantStudio 5 System, Thermo Fisher Scientific) with TaqMan Fast Advanced Master Mix (Thermo Fisher Scientific, 4444557) and TaqMan Gene Expression Assays (Thermo Fisher Scientific). The specific gene expression assays used are listed in Supplemental Table 1. Quantification of transcript levels was performed by the $2^{-\Delta \mathrm{CtT}}$ method, and expression levels for each gene were normalized to Ppia or Actb ( $\beta$-actin). RNA extracted from purified HSCs was normalized to Ppia or Pdgfrb.

\section{Glp2r full-length mRNA studies}

For human studies, the expression of full-length GLP2R and GAPDH transcripts was assessed by RT-PCR in total RNA from the human colon cancer cell line DLD-1, as previously confirmed (54), to stably express the human GLP-2R and in human liver total RNA purchased from the specified commercial sources (Lifetech, Agilent, Clontech). PCR products were analyzed by agarose gel electrophoresis followed by SYBR green staining and by Southern blot analysis using an internal GLP2R-specific ${ }^{32} \mathrm{P}$-labeled oligonucleotide probe. The presence of genomic DNA contamination in samples was monitored, in parallel, with control reactions in which the reverse transcriptase (RT) enzyme was omitted from the reaction mixture (RT-). Expression of mouse Glp2r and Gapdh transcripts was assessed following a similar protocol in mouse jejunum (positive control), liver, isolated hepatocytes, and purified HSCs. The human GLP2R PCR primer set is as follows: forward 5'-TTGTGAAGGTGCACGAGGAA-3' and reverse 5'-CACCCTAGATCTCACTCTCT-3'. Primers were amplified for 35 cycles at an annealing temperature of $60^{\circ} \mathrm{C}$. The product size of 1,687 bp spanned the entire GLP-2R open reading frame. The Gapdh PCR primer set is as follows: forward 5'-GACCACAGTCCATGACATCACT-3' and reverse 5'-TCCACCACCCTGTTGCTGTAG-3'. Primers were amplified for 25 cycles at an annealing temperature of $60^{\circ} \mathrm{C}$. The product size was $450 \mathrm{bp}$. This primer set is identical in human and mouse. The mouse Glp2r primer set is as follows: forward 
5'-GCCCAGTAGATGCAGAGAGG-3' and reverse 5'-AGTTGCCAAGCTGTGGTGAT-3'. Amplification was set for 37 cycles at an annealing temperature of $60^{\circ} \mathrm{C}$. The product size of $1,661 \mathrm{bp}$ spanned the entire GLP-2R open reading frame.

\section{Hepatocyte and hepatic NPC isolation}

Primary hepatocyte isolation was as previously described (55). For purification of the hepatic NPC fraction, the hepatocytes were depleted by 2 -minute centrifugation at $50 \mathrm{~g}$, and the supernatant containing NPCs was isolated and centrifuged for 5 minutes at $300 \mathrm{~g}$. The pellet was resuspended in hepatocyte wash medium (Thermo Fisher Scientific, 17704024). After 4 cycles of hepatocyte depletion, the NPC pellet was enriched in cells expressing macrophage and endothelial cell markers and depleted of albumin-expressing hepatocytes (Supplemental Figure 3A) and was collected for further analysis.

\section{Stellate cell isolation and FACS purification}

Isolation of stellate cells from mouse livers was performed following the protocol described in ref. 20. Briefly, the inferior vena cava was cannulated, the portal vein was resected, and the superior vena cava was clamped. Where appropriate, a segment of the left liver lobe was clamped prior to cannulation and resected for histology and mRNA/protein expression analyses. Following cannulation, the liver was perfused with a series of buffers containing EGTA, pronase, and collagenase; removed; and mechanically disrupted in a pronase plus collagenase mix at $37^{\circ} \mathrm{C}$. Stellate cells were then isolated by Nycodenz-based (Alere Technologies, 1002424) gradient isolation at $1380 \mathrm{~g}$ at $4^{\circ} \mathrm{C}$.

The stellate enriched fraction was removed, stained with the vital dye 7-aminoactinomycin D (7-AAD) (BD Pharmingen, 559925), and purified by flow cytometry performed by the flow cytometry core at The Centre for Phenogenomics using MoFlo Astrios EQ (Beckman Coulter) equipped with $405 \mathrm{~nm}, 488 \mathrm{~nm}, 561 \mathrm{~nm}$, and $640 \mathrm{~nm}$ lasers and running Summit v.6.2 software. Live stellate cells were identified by the following fluorescent signal combination: low 7-AAD (detector parameter 488-620/29 nm), high $405 \mathrm{~nm}$ violet laser autofluorescence (detector parameter 405-448/59 nm) and low $488 \mathrm{~nm}$ blue laser autofluorescence (detector parameter 488-513/26 nm). FACS gates were based on stellate cell autofluorescence in the violet range owing to cell-type specific vitamin A laden lipid droplets. Additional gating to remove cells based on autofluorescence in the FITC range enhanced purification from Kupffer cells and other minor contaminants. The postsorting violet ${ }^{+} \mathrm{FITC}^{-}$gate was confirmed to be highly enriched for HSC markers and depleted for markers of other hepatic cell populations (Figure 5D). Postsorting HSC viability was sampled frequently by 7-AAD and was reproducibly above $95 \%$.

\section{Flow cytometry for fractionation of hepatic NPC populations}

After collection of NPC-enriched fractions, cells were passed through a $40-\mu \mathrm{m}$ filter, collected by centrifugation at $300 \mathrm{~g}$, and resuspended in hepatocyte wash medium (Invitrogen-Gibco). Following 4 cycles of hepatocyte depletion, the NPC pellet was resuspended in FACS-sorting buffer (0.5 M EDTA, $25 \mathrm{mM}$ HEPES, and $1 \%$ FCS in PBS); incubated for 30 minutes at $4^{\circ} \mathrm{C}$ in antibody mixes based on CD-26-PE (Biolegend, 137804), CD-45-APC (Biolegend, 109814), and CD-31-FITC (Biolegend, 102406); and then washed and sorted. Flow cytometry was performed by the flow cytometry core at The Centre for Phenogenomics using equipment and software as detailed above.

\section{In vitro transcriptional response of IEGs in stellate cells}

IEG expression is activated by the stellate cell purification process (56) as well as by FBS that is typically added during cell sorting and recovery. Hence, we purified HSCs using standard protocols (20), and, following purification, pelleted the cells at $580 \mathrm{~g}$ and resuspended them in $1.5 \mathrm{ml}$ DMEM plus charcoal Dextran-treated (MilliporeSigma, C6241) bovine serum albumin (MilliporeSigma, A7030) BSA 1\% for 50 minutes at $37^{\circ} \mathrm{C}$. Following incubation, samples were divided into 3 equal parts and $10 \%$ FBS (positive control), GLP-2 (50 nM), or vehicle (PBS, in the same volume as GLP-2) was added. The dose of GLP-2 was based on previously established doses for direct induction of GLP-2R signaling in cells ex vivo (36).

To validate that incubation time sufficiently recovered IEG expression to levels amenable to activation, we tested whether the positive control cells, treated with 10\% FBS demonstrated expression at least 5\% 
higher than the PBS-treated negative control. For those samples, we then analyzed the expression in cells treated with $50 \mathrm{nM}$ GLP-2, as fold change from PBS-treated cells from the same batch.

\section{Statistics}

Statistical comparisons were made by 2-way ANOVA followed by a Tukey post hoc or by unpaired 2-tailed Student's $t$ test when only 2 conditions were compared. For in vitro incubation assays where attenuation of IEGs during recovery was variable, we compared control and treated samples using a 2-tailed ratio paired $t$ test. $P$ values of less than 0.05 were considered significant. Analysis was done using GraphPad Prism 7 or 8.2. No methods were used to determine whether the data met assumptions of the statistical approach. Statistical parameters can be found in the figure legends.

\section{Study approval}

All experiments involving mice were approved (animal use protocol approval 20-0045H) by the Animal Care and Use Subcommittee at the TCP at Mt. Sinai Hospital.

\section{Author contributions}

DJD designed the experiments, analyzed the data, and wrote the manuscript. SF, LLB, BY, DM, and EMV designed and executed the experiments, analyzed the data, and wrote the manuscript.

\section{Acknowledgments}

We thank Payal Shah (Lunenfeld Tanenbaum Research Institute, Mt. Sinai Hospital) for providing liver RNA analyzed from unpublished MCD experiments. SF has received research fellowship funding from the Canadian Pediatric Endocrine Group, Pediatric Endocrine Society, and the Hospital for Sick Children. DJD is supported by a Banting and Best Diabetes Centre-Novo Nordisk chair in Incretin Biology. EMV was supported by a Diabetes Canada fellowship. These studies were funded in part by operating grant support from the Canadian Institutes of Health Research 154321 and Shire-Takeda Inc.

Address correspondence to: Daniel J. Drucker, LTRI, Mt. Sinai Hospital, 600 University Avenue, Toronto, Ontario M5G1X5, Canada. Phone: 416.361.2661; Email: drucker@lunenfeld.ca.

1. Drucker DJ. Glucagon-like peptides. Diabetes. 1998;47(2):159-169.

2. Xiao Q, Boushey RP, Drucker DJ, Brubaker PL. Secretion of the intestinotropic hormone glucagon-like peptide 2 is differentially regulated by nutrients in humans. Gastroenterology. 1999;117(1):99-105.

3. Brubaker PL, Izzo A, Hill M, Drucker DJ. Intestinal function in mice with small bowel growth induced by glucagon-like peptide-2. Am J Physiol. 1997;272(6 Pt 1):E1050-E1058.

4. Drucker DJ, Erlich P, Asa SL, Brubaker PL. Induction of intestinal epithelial proliferation by glucagon-like peptide 2. Proc Natl Acad Sci USA. 1996;93(15):7911-7916.

5. Jeppesen PB, Gilroy R, Pertkiewicz M, Allard JP, Messing B, O'Keefe SJ. Randomised placebo-controlled trial of teduglutide in reducing parenteral nutrition and/or intravenous fluid requirements in patients with short bowel syndrome. Gut. 2011;60(7):902-914

6. Drucker DJ. The discovery of GLP-2 and development of teduglutide for short bowel syndrome. ACS Pharmacol Transl Sci. 2019;2(2):134-142.

7. Drucker DJ, Yusta B. Physiology and pharmacology of the enteroendocrine hormone glucagon-like peptide-2. Annu Rev Physiol. 2014;76:561-583.

8. Yusta B, et al. Glucagon-like peptide-2 promotes gallbladder refilling via a TGR5-independent, GLP-2R-dependent pathway. Mol Metab. 2017;6(6):503-511

9. Gether IM, Nexøe-Larsen C, Knop FK. New avenues in the regulation of gallbladder motility-implications for the use of glucagon-like peptide-derived drugs. J Clin Endocrinol Metab. 2019;104(7):2463-2472.

10. Yusta B, Matthews D, Koehler JA, Pujadas G, Kaur KD, Drucker DJ. Localization of glucagon-like peptide-2 receptor expression in the mouse. Endocrinology. 2019;160(8):1950-1963.

11. El-Jamal N, et al. Glugacon-like peptide-2: broad receptor expression, limited therapeutic effect on intestinal inflammation and novel role in liver regeneration. Am J Physiol Gastrointest Liver Physiol. 2014;307(3):G274-G285.

12. Halpern KB, et al. Single-cell spatial reconstruction reveals global division of labour in the mammalian liver. Nature. 2017;542(7641):352-356.

13. Taher J, Baker C, Alvares D, Ijaz L, Hussain M, Adeli K. GLP-2 dysregulates hepatic lipoprotein metabolism, inducing fatty liver and vldl overproduction in male hamsters and mice. Endocrinology. 2018;159(9):3340-3350.

14. Cani PD, et al. Changes in gut microbiota control inflammation in obese mice through a mechanism involving GLP-2-driven improvement of gut permeability. Gut. 2009;58(8):1091-1103.

15. Buchman AL, Naini BV, Spilker B. The differentiation of intestinal-failure-associated liver disease from nonalcoholic fatty liver 
and nonalcoholic steatohepatitis. Semin Liver Dis. 2017;37(1):33-44.

16. Javid PJ, Oron AP, Duggan CP, Squires RH, Horslen SP, Pediatric Intestinal Failure Consortium. The extent of intestinal failure-associated liver disease in patients referred for intestinal rehabilitation is associated with increased mortality: an analysis of the Pediatric Intestinal Failure Consortium database. J Pediatr Surg. 2018;53(7):1399-1402.

17. Baldassano S, Amato A, Rappa F, Cappello F, Mulè F. Influence of endogenous glucagon-like peptide-2 on lipid disorders in mice fed a high-fat diet. Endocr Res. 2016;41(4):317-324.

18. Matikainen $\mathrm{N}$, et al. Vildagliptin therapy reduces postprandial intestinal triglyceride-rich lipoprotein particles in patients with type 2 diabetes. Diabetologia. 2006;49(9):2049-2057.

19. Hsieh $\mathrm{J}$, et al. The glucagon-like peptide 1 receptor is essential for postprandial lipoprotein synthesis and secretion in hamsters and mice. Diabetologia. 2010;53(3):552-561

20. Mederacke I, Dapito DH, Affò S, Uchinami H, Schwabe RF. High-yield and high-purity isolation of hepatic stellate cells from normal and fibrotic mouse livers. Nat Protoc. 2015;10(2):305-315.

21. Yusta B, et al. ErbB signaling is required for the proliferative actions of GLP-2 in the murine gut. Gastroenterology. 2009;137(3):986-996.

22. De Minicis S, et al. Gene expression profiles during hepatic stellate cell activation in culture and in vivo. Gastroenterology. 2007;132(5):1937-1946.

23. Baroni GS, et al. Interferon gamma decreases hepatic stellate cell activation and extracellular matrix deposition in rat liver fibrosis. Hepatology. 1996;23(5):1189-1199.

24. Mederacke I, et al. Fate tracing reveals hepatic stellate cells as dominant contributors to liver fibrosis independent of its aetiology. Nat Commun. 2013;4:2823.

25. Kristensen DB, et al. Proteome analysis of rat hepatic stellate cells. Hepatology. 2000;32(2):268-277.

26. Domenicali M, et al. A novel model of CCl4-induced cirrhosis with ascites in the mouse. J Hepatol. 2009;51(6):991-999.

27. Perepelyuk M, et al. Hepatic stellate cells and portal fibroblasts are the major cellular sources of collagens and lysyl oxidases in normal liver and early after injury. Am J Physiol Gastrointest Liver Physiol. 2013;304(6):G605-G614.

28. Kelly DA. Intestinal failure-associated liver disease: what do we know today? Gastroenterology. 2006;130(2 Suppl 1):S70-S77.

29. Yano K, et al. Novel effect of glucagon-like peptide-2 for hepatocellular injury in a parenterally fed rat model of short bowe syndrome. Pediatr Surg Int. 2019;35(12):1345-1351.

30. Meier JJ, et al. Glucagon-like peptide 2 stimulates glucagon secretion, enhances lipid absorption, and inhibits gastric acid secretion in humans. Gastroenterology. 2006;130(1):44-54.

31. Hsieh J, et al. Glucagon-like peptide-2 increases intestinal lipid absorption and chylomicron production via CD36. Gastroenterology. 2009;137(3):997-1005.e1.

32. Hsieh J, et al. Glucagon-like peptide 2 (GLP-2) stimulates postprandial chylomicron production and postabsorptive release of intestinal triglyceride storage pools via induction of nitric oxide signaling in male hamsters and mice. Endocrinology. 2015;156(10):3538-3547.

33. Schwartz LK, et al. Long-term teduglutide for the treatment of patients with intestinal failure associated with short bowel syndrome. Clin Transl Gastroenterol. 2016; 7:e142.

34. Choi WM, et al. Glutamate signaling in hepatic stellate cells drives alcoholic steatosis. Cell Metab. 2019;30(5):877-889.e7.

35. Dash S, Xiao C, Morgantini C, Connelly PW, Patterson BW, Lewis GF. Glucagon-like peptide-2 regulates release of chylomicrons from the intestine. Gastroenterology. 2014;147(6):1275-1284.e4.

36. Yusta B, et al. Identification of glucagon-like peptide-2 (GLP-2)-activated signaling pathways in baby hamster kidney fibroblasts expressing the rat GLP-2 receptor. J Biol Chem. 1999;274(43):30459-30467.

37. Yusta B, et al. Enteroendocrine localization of GLP-2 receptor expression in humans and rodents. Gastroenterology. 2000;119(3):744-755.

38. Marcher AB, et al. Transcriptional regulation of hepatic stellate cell activation in NASH. Sci Rep. 2019;9(1):2324.

39. Machado MV, et al. Mouse models of diet-induced nonalcoholic steatohepatitis reproduce the heterogeneity of the human disease. PLoS One. 2015;10(5):e0127991.

40. Yoshimoto S, et al. Obesity-induced gut microbial metabolite promotes liver cancer through senescence secretome. Nature. 2013;499(7456):97-101.

41. Brun P, Castagliuolo I, Pinzani M, Palù G, Martines D. Exposure to bacterial cell wall products triggers an inflammatory phenotype in hepatic stellate cells. Am J Physiol Gastrointest Liver Physiol. 2005;289(3):G571-G578.

42. Chimerel C, Emery E, Summers DK, Keyser U, Gribble FM, Reimann F. Bacterial metabolite indole modulates incretin secretion from intestinal enteroendocrine L cells. Cell Rep. 2014;9(4):1202-1208.

43. Nguyen AT, et al. Lipopolysaccharides-mediated increase in glucose-stimulated insulin secretion: involvement of the GLP-1 pathway. Diabetes. 2014;63(2):471-482.

44. Lebrun LJ, et al. Enteroendocrine L cells sense LPS after gut barrier injury to enhance GLP-1 secretion. Cell Rep. 2017;21(5):1160-1168

45. Thomas C, et al. TGR5-mediated bile acid sensing controls glucose homeostasis. Cell Metab. 2009;10(3):167-177.

46. Müller TD, et al. Glucagon-like peptide 1 (GLP-1). Mol Metab. 2019;30:72-130.

47. Maeso-Díaz R, et al. Effects of aging on liver microcirculatory function and sinusoidal phenotype. Aging Cell. 2018;17(6):e12829.

48. Shi X, et al. Central GLP-2 enhances hepatic insulin sensitivity via activating PI3K signaling in POMC neurons. Cell Metab. 2013;18(1):86-98

49. Lee SJ, et al. Disruption of the murine Glp2r impairs Paneth cell function and increases susceptibility to small bowel enteritis. Endocrinology. 2012;153(3):1141-1151.

50. Softic S, et al. Dietary sugars alter hepatic fatty acid oxidation via transcriptional and post-translational modifications of mitochondrial proteins. Cell Metab. 2019;30(4):735-753.e4.

51. Clapper JR, et al. Diet-induced mouse model of fatty liver disease and nonalcoholic steatohepatitis reflecting clinical disease progression and methods of assessment. Am J Physiol Gastrointest Liver Physiol. 2013;305(7):G483-G495. 
52. Scholten D, Trebicka J, Liedtke C, Weiskirchen R. The carbon tetrachloride model in mice. Lab Anim. 2015;49(1 Suppl):4-11.

53. Folch J, Lees M, Sloane Stanley GH. A simple method for the isolation and purification of total lipides from animal tissues. $J$ Biol Chem. 1957;226(1):497-509.

54. Koehler JA, Harper W, Barnard M, Yusta B, Drucker DJ. Glucagon-like peptide-2 does not modify the growth or survival of murine or human intestinal tumor cells. Cancer Res. 2008;68(19):7897-7904.

55. Flock G, Baggio LL, Longuet C, Drucker DJ. Incretin receptors for glucagon-like peptide 1 and glucose-dependent insulinotropic polypeptide are essential for the sustained metabolic actions of vildagliptin in mice. Diabetes. 2007;56(12):3006-3013.

56. van den Brink SC, et al. Single-cell sequencing reveals dissociation-induced gene expression in tissue subpopulations. Nat Methods. 2017;14(10):935-936. 